\title{
Spatial numerical estimates within proprioceptive recalibration
}

\author{
Thiago Gomes de Castro*, Marcelle Matiazo Pinhatti, Clarissa Pinto Pizarro de Freitas, and William Barbosa Gomes
}

Federal University of Rio Grande do Sul (Brazil).

\begin{abstract}
Título: Estimaciones numéricas espaciales en la recalibración propioceptiva.

Resumen: La literatura en psicología cognitiva ha enfatizado que la posición del cuerpo en el espacio y los patrones de búsqueda visual de estímulos son variables cruciales para explicar la capacidad de estimar distancias numéricamente. En este artículo, hemos probado la hipótesis de que la recalibración de la propiocepción interfiere en la capacidad de estimar numéricamente el espacio peri-personal fijo. El paradigma experimental Rubber Hand Illusion (RHI) se aplicó como herramienta para manipular temporalmente el sentido de propiocepción en la mano derecha del participante. Se pidió a diecisiete estudiantes universitarios que, dentro de la RHI, estimasen señales espaciales fijadas horizontalmente antes y después de dos condiciones de estimulación táctil (estimulaciones síncronas $\mathrm{x}$ asíncronas). Los resultados evidenciaron que la recalibración proprioceptiva de la mano fue temporalmente alterada por ambos patrones de estimulación. Sin embargo, los efectos de estimar las señales fijas numéricamente en dirección de la línea media del cuerpo sólo fueron observados consistentemente en la condición de estimulación síncrona.

Estos resultados sugieren que la estimación numérica de señales fijas peripersonales está fuertemente asociadas con la recalibración proprioceptiva, corroborando la literatura sobre integración multisensorial de la percepción.

Palabras clave: propriocepción; estimación numérica; representación del cuerpo; rubber hand illusion; procesos cognitivos.
\end{abstract}

\section{Introduction}

The Rubber Hand Illusion (RHI) experimental paradigm has brought forward a distinctive way of investigating the dynamics of proprioception and the embodiment of out-ofbody objects. The paradigm has influenced neuroscience research specially by drawing different modes of investigation for multisensory processes. Botvinick and Cohen (1998) created the RHI firstly to evaluate the intermodal proprioceptive integration in self-attribution of phantom limbs. The perceptual illusion is produced by continually stroking synchronously one of participant's real hand and a prosthetic rubber hand positioned in front of the subject. RHI recalibrates the proprioception of the own hand to the fake hand as a consequence of the distortion in the interaction of vision, touch, and sense of body position in space. Overall $80 \%$ of participants feel the rubber hand as its own hand after approximately 15-30 seconds of synchronous stroking (Ehrsson, Holmes, \& Passingham, 2005). Research has evidenced that proprioceptive sense of limb position and subjective feeling of ownership of the fake hand are central variables manipulated during RHI experiment (Fiorio et al., 2014).

The illusion is conditioned by a synchronous stroking between the real hand and the rubber hand (Ehrsson, Spence,

* Correspondence address [Dirección para correspondencia]:

Thiago Gomes de Castro. Federal University of Rio Grande do Sul, Rua

Ramiro Barcelos, n²600 - Instituto de Psicologia - Sala 123 / 90035-

003 / Porto Alegre - RS (Brazil). E-mail: thiago.cast@gmail.com
Abstract: Research in cognitive psychology has emphasized that the body's position in space and patterns of visual searching for stimuli are crucial variables to explain the ability to estimate distances numerically. In this paper, we tested the hypothesis that proprioception recalibration interferes in the ability to numerically estimate fixed peri-personal space. The Rubber Hand Illusion (RHI) experimental paradigm was applied as a tool to temporally manipulate the sense of proprioception in participant's right hand. Seventeen college students were asked to estimate horizontal fixed spatial cues before and after two conditions of tactile stimulation within RHI (synchronous versus asynchronous stroking). Results evidenced that proprioceptive recalibration of the hand were temporally altered by both stroking patterns. However, the effects of numerically estimate fixed horizontal cues towards the body midline were only consistently observed in the synchronous stroking condition. These findings suggest that numerical estimates of peri-personal fixed cues are strongly associated with proprioceptive recalibration, corroborating the literature on multisensory integration of perception.

Key words: proprioception; spatial estimates; body representation; rubber hand illusion; cognitive processes.
\& Passingham, 2004). Asynchronous stroking diminishes the effect or even eliminates the proprioceptive illusion in healthy groups (Kaplan, Enticott, Hohwy, Castle, \& Rossell, 2014). The asynchronous effect is explained as a consequence of the delay between feeling the touch and seeing the touch (Shimada, Suzuki, Yoda, \& Hayashi, 2014). However, the asynchronous effect, in order to break the illusion, must respect specific time limits. Bekrater-Bodmann et al. (2014) showed that asynchronies of up to $300 \mathrm{~ms}$ between seeing the touch and feeling the touch are not sufficient to break the proprioceptive illusion generated by regular synchronous RHI. However, if the asynchronies reach intervals greater than $600 \mathrm{~ms}$, it is observed a sharp drop in brain activity related to the illusion. At the same time asynchronies greater than $600 \mathrm{~ms}$ decrease ownership experience of the rubber hand.

Recent studies on body perception have explored the effect of distorted visual feedback of the real hand on horizontal proprioceptive estimates of the hand (Clayton, Cressman, \& Henriques, 2013; Mostafa, Salomonczyk, Cressman, \& Henriques, 2014; Salomonczyk, Cressman, \& Henriques, 2011). Results showed strong interaction between proprioceptive feeling of the body and its surrounding space perception, especially in the estimation of limb distances in an horizontal plane. The perception of altered body space, as suggested in RHI, refers to the relationship between spatiality and cognitive skills of recognition of stimuli in space. Tanaka, Worringham and Kerr (2009) suggested that the interaction between vision and proprioception is critical for 
accurate representation of online target-effectors spatial estimates. Such kinematic sensorimotor representations serve as a cognitive function of target position prediction. In RHI the recognition of the tactile sensation in a prosthesis located in a distinct visual position regarding the natural location of the real hand indicates a temporary change in the upper limbs location scheme. In this direction, it is interesting to investigate how proprioceptive recalibration of effectors interfere over the ability to assess and estimate short distances around the body, the peri-personal space (Cardinali, Brozzoli, \& Farnè, 2009).

One way to evaluate space estimates is by measuring distance predictions via numerical representation. The intricate relationship between spatial representations and numerical representations has been consistently referred in the literature (Arend, Naparstek, \& Henik, 2013; DeHevia, Vallar, \& Girelli, 2008; Van Dyck \& Fias, 2011). In the last two decades, one of the most accepted hypothesis for the correlation between spatial representations and numerical spatial encoding is the Mental Number Line hypothesis (Dehaene, 1992; Dehaene \& Cohen, 1997; Dehaene, Piazza, Pinel, \& Cohen, 2003). The hypothesis sustains that the spatial representation in humans occurs as a nonverbal mental number line, which would be a continuous logarithmic compression that provides numerical magnitude information to represent distances (Dehaene et al., 2003). In western individuals the predominance of this representation occurs in a horizontal axis, as a growing number line from left to right on the visual field horizon (Holmes \& Lourenco, 2012).

In the context of RHI experiment, the relation between bodily spatial perception and spatial numerical representations was initially demonstrated in a research by Tsakiris and Haggard (2005). The researchers indicated a correlation between the proprioceptive recalibration provided by RHI and the corresponding spatial numerical estimate reported by participants regarding the position of their index finger. In this research participant's had to judge where they felt their index finger after four minutes of stroking. Fiorio et al. (2011) also used spatial numerical estimates to assess proprioceptive recalibration in the RHI. Participants had to refer the felt index finger position after stimulation. The results showed that assessments of the RHI effect were in line with the estimates reported in the numerical spatial judgment. The experiments conducted by Tsakiris and Haggard (2005) and Fiorio et al. (2011) have demonstrated the use of numerical scales as a verification index of proprioceptive recalibration. Both studies used the feeling of limb/index position as a target of spatial estimates. These results are interesting because the estimates in post-stimulation corroborated the proprioceptive drift caused by RHI. However, measurement of felt position after illusion represents a different criterion of evaluation from the judgment of fixed stimuli in the peripersonal space. Felt position would be related to an index of proprioceptive drift, but not to the modification of peripersonal perception.
Few studies have explored the direct relationship between the proprioceptive modification produced by RHI and the perception of fixed distances relative to the body. Dempsey-Jones and Kritikos (2012) investigated perceptual judgements of fixed limb position during RHI and found no effects of functional hemispheric asymmetry (handedness) on the recalibration processing of body-space. Nevertheless, RHI was effective in modulating the perceptual judgments by means of attention to hand-space. Also Butz, Kutter and Lorenz (2014) found that the proprioceptive drift not only adjust the perception of the fixed hand in body-relative space, but includes modification of perception in the relative elbow. Both studies explored the relation between proprioceptive drift and estimates of fixed body-space stimuli, but none of them evaluated these estimates in terms of numerical estimation judgements.

In the present experiment we sought to explore this specific relationship between proprioceptive recalibration and fixed body-space numerical judgments. Therefore the research aimed to investigate pre and post numerical estimates towards fixed real index finger, using different patterns of stimulation in the context of RHI. The main purpose of the study was to verify if the temporary illusion produced by the RHI modifies the sense of spatial numerical estimates in relation to the stable position of the real right index finger.

\section{Method}

\section{Participants}

Seventeen subjects ( $59 \%$ women), with mean age 24 years $(S D=4.63$, ranging from 19 to 28 years old), normal or corrected to normal vision, all right-handed were initially selected. The selection was incidental and took place at a University in Southern Brazil. All participants were students at different faculties in the University. The research was approved by the Research Ethics Committee of the local University. Each participant provided informed consent. Participants were informed about the nature and the purpose of the study and were assured that information provided during the experiment would be kept confidential. The descriptive analysis adopted the outlier's exclusion criteria suggested by Reuschel, Drewing, Henriques, Rösler and Fiehler (2010) in experiments with visual spatial estimates. Results that exceeded the average estimates in two standard deviations were excluded from the analysis. Following this criteria, two specific estimates across all trials were identified and replaced by the average estimate values of their respective condition and estimation task. We chose this amount of participants as our target sample size, because past studies of rubber hand illusion drawn conclusions from 15-30 participants sample size (e.g., Bekrater-Bodman et al., 2014; Butz et al., 2014; Constantini \& Haggard, 2007). 


\section{RHI Setting}

Participants were tested individually. They sat in a chair in front of a rectangular table. A prosthetic right rubber hand was located opposite to the participant on the table. A partition occluded participant real right hand (see Figure 1). The partition aimed to obstruct the participant's view of the stimulated real right hand which was placed on the table in a predefined marking. Two right hand prostheses were used in the experiment, one similar to a male hand and the other to a female hand. The prostheses were made of a silicone fabric with the purpose to resemble human skin texture. The distance between the real right index finger and the prosthetic index finger was standardized in $26 \mathrm{~cm}$, and from the unstimulated real left index finger to the real right index finger was $60 \mathrm{~cm}$, as indicated by Ehrsson et al. (2008). Two brushes with identical thick tip were used for stimulation. A black cotton cloth was placed on the right arm of participants in order to strengthen the discontinuity between the chest and the real right hand, and a second black cotton cloth was used to hide the forearm of the prosthesis. Two $60 \mathrm{~cm}$ wooden metric boards were used, each with two metric tapes glued on front and back sides (four rulers in total): one ruler for each stimulation condition $\mathrm{x}$ estimate trial (2X2). The front part of the board was intended for pre-stimulation estimate (PrSE) and the back part of the board for post-stimulation estimate (PoSE). The four rulers had an ascending order from left to right, with the same numerical range, but with different numbering between each other. A stopwatch and two voice recorders were also used, one to record the voice of the participants, and another to produce a rapid constant sound pulse after the end of the two minutes of stimulation (signaling the participant the interval for the PoSE).
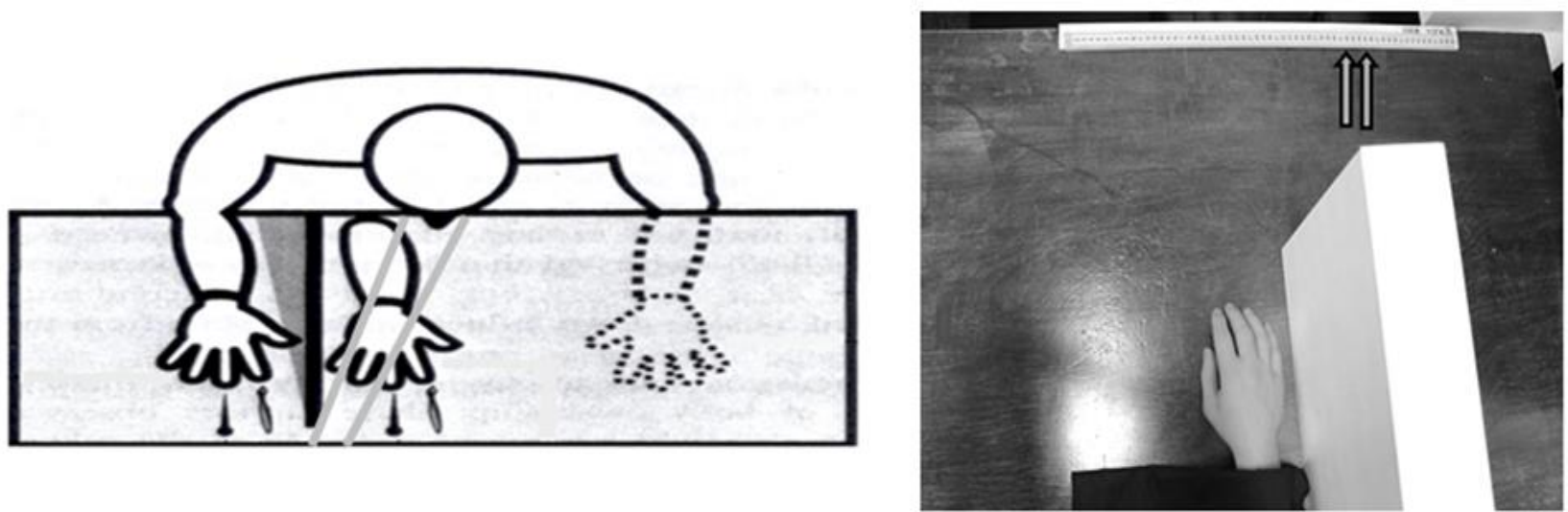

Figure 1. RHI setting / aerial photo of the setting with the prosthesis: left arrow (fixed limit range of vision), right arrow (fixed position of the real right index finger).

\section{RHI Procedures}

Initially the participants sat in front of a table and positioned the left and right index fingers on the markings predefined. Then the experimenter placed the partition between the participant's right forearm and the prosthesis. The participant was instructed to centralize his body midline according to a marking spot centralized on the base of the table. Once in position, participants were requested to name the cutpoint limit number of his visual range towards the right side on the ruler. The previously standardized maximum range for all participants should be $42 \mathrm{~cm}$ in a range of 0 to $60 \mathrm{~cm}$. This cut-point configuration was standardized for the sample by adjusting the partition in the pre-experiment phase. After informing the limit number on the visual range field, participants were asked to state which number on the ruler corresponded to the position of his right index finger (PrSE). In this statement participants always produced a supposition estimation of his right index finger position, given that they could not visually reach the exact fixed position of the real index finger $(45 \mathrm{~cm}), 3 \mathrm{~cm}$ away from their visual cut point.
Once the pre-stimulation estimate (PrSE) was performed, the tactile stimulation between the real right hand and the prosthesis began. This procedure was divided in two blocks: synchronous stimulation (Condition A) and asynchronous stimulation (Condition $\mathrm{B}$ ), both conditions were randomized (A-B/B-A) between participants. Participants were instructed to visually target the rubber prosthesis during the entire period of stimulation, which took two minutes per block. The tactile stimulation was produced at a rate of one pulse per second $(1 \mathrm{~Hz})$, synchronized in condition A and with 1x1 second non-synchronized intervals in condition B.

In the initial 30 seconds of stimulation, the experimenter applied the tactile stroking only in the index fingers of the real and prosthetic hands. After this interval, the stimulation then alternated between the middle, ring and little fingers, consecutively, and then restarted in the index finger. An average of eight pulses of stimulation per finger was respected in this phase. The experimenter applied the stimulus between the hand and the prosthesis equally for each finger, regardless of stimulation condition, varying only the stimulus sync time. The tactile stimulus with the brush was applied contin- 
uously from the base of the metacarpal bone to the bodies of the distal phalanges. After two minutes of stimulation, an acoustic pulse was issued to signal that participant should provide new estimates of distance on the position of his real index finger (PoSE). A different side of the ruler was used for each estimate in order to prevent that the participant would get habituated with the previously measured value. After each stimulation condition, participants answered the Proprioceptive Distortion Questionnaire - PDQ (Botvinick \& Cohen, 1998), composed of nine items regarding the experience within RHI setting.

\section{Design}

The experiment employed a two factor within-subject design with factors stroking condition (Synchronous vs. Asynchronous stroking) and estimation task (PrSE vs. PoSE stimulation estimate). The dependent variables were poststimulation estimates (PoSE), difference estimate coefficient (DEC), and PDQ scores. The formula for the proprioceptive changes in the DEC is reached by the difference between measurements in the pre- and post-stimulus considering each condition. In this computation the PrSE works as a relative zero to the PoSE, meaning the distance of deviation from that zero. PrSE, PoSE and DEC include the direction of the estimate, if to the left $(+)$ or to the right $(-)$ of the real right index finger.

\section{Statistical Analysis}

In order to analyze the effect of experimental stroking condition (Synchronous vs. Asynchronous stroking) over differences in the estimation task (PrSE vs. PoSE), 2 x 2 analysis of variance (ANOVA) with two within-subjects factors were performed. The factors were (a) the condition of stroking (synchronous vs. asynchronous) and (b) the moment of estimation (PrSE vs. PoSE). Furthermore differences within each experimental stroking condition considering the moment of estimation were analyzed through $T$ test analyses. The differences of DEC and PDQ according to the experimental stroking condition were also tested through $T$ test analysis. The correlations between the PrSE and PoSE in Synchronous (PrSES and PoSES) and Asynchronous Condition (PrSEA and PoSEA), DEC in Synchronous (DECS) and Asynchronous Condition (DECA) were investigated. Because the data were non-normally distributed, Spearman's rho correlations were performed. No multicollinearity was found. Effect size (Cohen's $d, \eta^{2}$ and $r_{\ell}{ }^{2}$ ) were evaluated for all tests. The analyses above cited were performed in the Statistical Package for the Social Sciences - Version 21.0 (SPSS 21.0).

\section{Results}

The deviation pattern of estimation without proprioceptive illusion (PrSE) was different for both stroking conditions (see Table 1). The magnitude of the deviation patterns between conditions was high $(d=0.84)$. These pre-stimulation estimates occurred within $2 \mathrm{~cm}$ limits of deviation from the real right index finger. Furthermore no effect of sequence (A-B/B-A) was found between conditions, disregarding any possible explanation of superposing effect between conditions. When comparing the post-illusion estimates alone (PoSE) between conditions no effect was found. Nevertheless, deviations in PoSE at synchronous condition occurred beyond $2 \mathrm{~cm}$ whereas deviations in PoSE at asynchronous condition occurred under $2 \mathrm{~cm}$.

This regular calculation provides only a pattern of general deviation, disregarding important information about the direction deviation pattern and the real distance between base line estimation (PrSE) and post stroking estimation (PoSE) for each condition. Overall, the average of estimated deviations in PoSE is higher than the overall average in PrSE $[t(33)=-2.020, p \leq .05$, Cohen's $d=.378]$, indicating effect of stroking manipulation regardless of stroking pattern. However, considering the DEC calculation, which provides an accurate distance value between PrSE and PoSE for each stroking condition, it was observed a consistent difference between estimation tasks $[t(16)=2.802, p \leq .05$, Cohen's $d=$ .673], with a larger distance amplitude DEC for synchronous stroking.

Table 1. Longitudinal analysis of PrSE and PoSE in Synchronous (PrSES and PoSES) and Asynchronous Condition (PrSEA and PoSEA), DEC in Synchronous (DECS) and Asynchronous Condition (DECA), levels of PDQ in Synchronous (PDQS) and Asynchronous Condition (PDQA).

\begin{tabular}{lcccccc} 
& Synchronous & Asynchronous & Comparisons & $F(d f)$ & $\boldsymbol{\omega}^{2}$ & $t(d f)$ \\
\hline PrSE & $-.3(3.6)$ & $1.5(2.8)$ & Synchronous x Asynchronous & $1.96(1,16)$ & .11 & \\
PoSE & $2.2(4.4)$ & $1.8(3.8)$ & PrSE x PoSE & $2.94(1,16)$ & .15 & \\
& & & Interaction & $4.97^{*}(1,16)$ & .24 & \\
& & & (Condition x Time) & & $2.2^{*}(16)$ & 0.53 \\
& & & PrSES x PoSES & $.40(16)$ & 0.10 \\
& & & PrSEA x PoSEA & $-3.3^{*}(16)$ & 0.84 \\
& & & PrSES x PrSEA & $.5(16)$ & 0.12 \\
DEC & $2.9(4.5)$ & $.3(4.5)$ & PoSES x PoSEA & & $2.8^{*}(16)$ & 0.67 \\
PDQ & $14.2(5.3)$ & $8.9(4.5)$ & DECS x DECA & & $3.5^{*}(16)$ & 0.84 \\
\hline
\end{tabular}

Note: $* \leq .05$. 
The specific effects of experimental stroking condition (Synchronous vs. Asynchronous) over differences in the estimation task (PrSE vs. PoSE) were analyzed through $2 \times 2$ ANOVA with two within-subjects factors. There was a significant effect due the interaction of the two factors $[F(1,16)$ $=4,97, p \leq .05$, partial $\left.\eta^{2}=.24\right]$. However, it was not observed a main effect due the moment of the estimation task and the condition of stroking. In order to evaluate the effects of the interaction in more detail, $T$ tests were performed be- tween estimate trials. It was observed a consistent trend in synchronous condition towards larger amplitude of deviation in PoSE compared to PrSE. A moderate effect size $(d=$ 0.53) was obtained. The same comparison between PrSE and PoSE in asynchronous stroking did not show effect. The distribution of estimate differences between the two conditions is represented in Figure 2, taking PrSE value as a relative zero. Figure 2 also illustrates an estimative directional trend towards the rubber hand position.

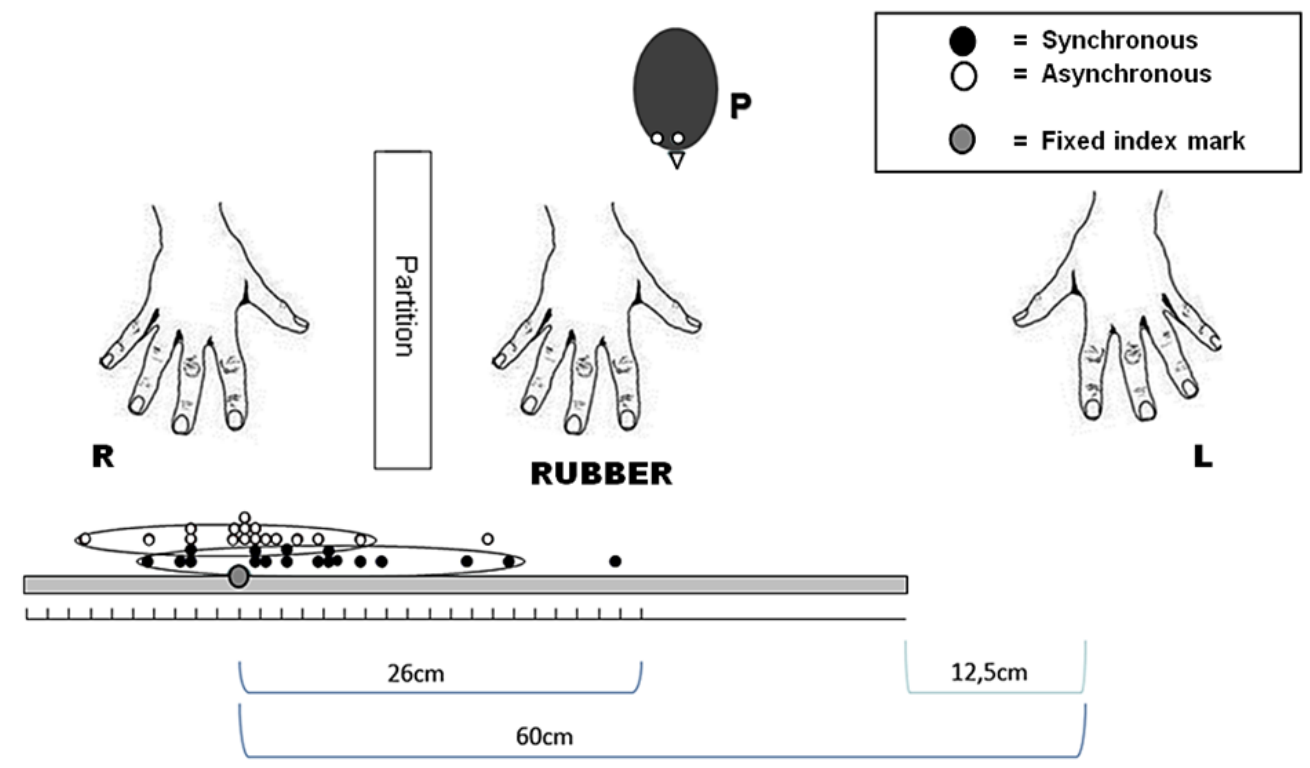

Figure 2. Illustration of directional deviation in PoSE from relative zero (PrSE).

Correlational analyses evidenced that PrSE's between synchronous and asynchronous condition were positively associated $\left(r_{\varrho}{ }^{2}=.65\right.$, large effect size). Post stimulation estimates (PoSE) were also correlated between conditions $\left(r_{\ell}{ }^{2}=\right.$ .49, moderate effect size). PrSE correlated with PoSE in asynchronous condition $\left(r_{\ell}{ }^{2}=.31\right.$, weak effect size $)$, but the same correlation was not observed in the synchronous condition. PoSE in synchronous condition were correlated with DEC in synchronous $\left(r_{\ell}{ }^{2}=.44\right.$, moderate effect size $)$ and asynchronous condition $\left(r_{\ell}{ }^{2}=.46\right.$, moderate effect size). PoSE in asynchronous condition were positively related to DEC in the same condition $\left(r_{\ell}^{2}=.49\right.$, moderate effect size). DEC in synchronous were positively associated with DEC in asynchronous condition $\left(r_{\varrho}{ }^{2}=.31\right.$, weak effect size) (see Table 2). Finally, no correlation was observed between PDQ scores and estimation tasks, neither between PDQ responses in both stroking conditions.

The intense change observed between PrSE and PoSE in the synchronous stroking condition was supported by the reports in the PDQ. The first three items of the instrument, which assess the illusory experience created by the experiment, reached higher averages than the average for the same three items regarding the asynchronous condition $[t(16)$ $=3,497, p<.001$, Cohen's $d=1.073$ ]. The large size effect signals powerfull subjective feeling of proprioceptive distortion induced by the synchronous stroking condition. During the synchronous stimulation participant's often reported experience of ownership of the rubber hand (e.g. "That's my hand!" or "It feels like this one [rubber hand] is my hand").

Table 2. Spearman's Rho Correlations Between the PrSE and PoSE in Synchronous (PrSES and PoSES) and Asynchronous Condition (PrSEA and PoSEA), DEC in Synchronous (DECS) and Asynchronous Condition (DECA).

\begin{tabular}{llllll}
\hline Constructs & 1 & 2 & 3 & 4 & 5 \\
\hline 1. PrSES & & & & & \\
2. PoSES & .34 & & & & \\
3. PrSEA & $.81^{* *}$ & .38 & & & \\
4. PoSEA & $.53^{*}$ & $.70^{* *}$ & $.56^{*}$ & & \\
5. DECS & -.36 & $.66^{* *}$ & -.22 & .22 & \\
6. DECA & .05 & $.68^{* *}$ & -.03 & $.70^{* *}$ & $.56^{*}$ \\
\hline Note: ${ }^{*} p<.05,{ }^{* *} p<.001$. & & & &
\end{tabular}

Given the proprioceptive changes between PrSE and PoSE, specially in synchronous condition, a topographical analysis of the directional patterns of deviation was conducted. The results showed a consolidated trend, in both conditions, to estimate in PoSE numbers closer to the 
rubber prosthesis (63\% of all estimates). The opposite direction was observed in only $24.7 \%$ in PoSE, when participants estimated more to the right than the fixed position of the real right index finger. There were also cases where there was no estimate variation between pre and post stimulation, these situations were considered perfect estimations $(12.3 \%)$. These directional estimative patterns were relatively homogeneous between stroking conditions, without statistical differences. However, as described above, the amplitude values of estimative difference between pre and post were higher for synchronous condition, and in most instances of this condition (75\%) towards the rubber prosthesis in PoSE.

\section{Discussion}

The findings suggest two main effects of the illusion. First that synchronous tactile stimulation interferes in the ability to estimate numerically fixed peri-personal distances. Secondly, that synchronous tactile stimulation produces subjective perception of ownership for the rubber hand prosthesis (PDQ results). In general, the differential effect of synchronous stroking in producing the illusion has been consistently reported in the literature (Ehrsson et al., 2004; Kaplan et al., 2014). However there have been no consistent reports of differential effect of synchronous stroking in numerical estimates of fixed body position, which precludes possibilities to discuss the relationship between high order cognitive processes and proprioceptive distortion.

We found an overall prevalence of estimates towards the body midline (Rubber Hand direction) in PoSE, with greater spatial amplitude between PrSE and PoSE for the synchronous stroking condition. The literature on proprioceptive estimates of upper limbs reports a normal tendency to deviate around $2 \mathrm{~cm}$ to the left or to the right of the actual position of the real limbs (Jones, Cressman, \& Henriques, 2010). These deviation patterns occur when measuring estimates of rested arms on a flat surface without any interference of illusion, a similar condition compared to the setting of RHI in pre-stimulation. The prevalence of these estimation errors is typically convergent to the right of the right arm or to the left of the left arm. Therefore, directed to the opposite side of the body midline. This observation contrasts to what has been found in the present research. Here, the average of estimates deviation, without the influence of proprioceptive illusion (PrSE), was close to that reported by Jones et al. (2010), regardless of stroking pattern. However it stood up the effect of tactile stimulation in post illusion estimates (PoSE). The observed tendency to estimate fixed distances towards the prosthesis, after the stimulation, confirms the hypothesis of interference of the RHI in the recalibration of body scheme in proximal spaces (Botvinick \& Cohen, 1998). This estimation trend towards the midline was especially robust as a response to the synchronous stroking. Correlational analyses corroborated the effect of stimulation in the estimation tasks, indicating moderate effect sizes between PoSE and DEC, for both stimulation conditions, and no effect between PrSE and DEC's. This selective association between PoSE's and DEC's evidence an effect of stimulation in the high amplitude variation observed in PoSE's compared to PrSE's.

It must be emphasized that the effect of the illusion in the RHI has been traditionally assessed through estimate measurements in relation to the felt position of the recalibrated limb, but not through numerical estimates of fixed bodily spaces. This means that the effect of proprioceptive recalibration is usually investigated within the context of subjective effector recalibration. The evaluation of perceived proprioceptive recalibration in the RHI has trusted so far in blind reaching of perceived stimulated hand by means of contralateral hand (e.g., Ehrsson et al., 2008) or numerical designation of felt index finger location during stimulation (e.g., Fiorio et al., 2011). Our results add a new finding regarding the correlation between proprioceptive distance predictions and spatial judgements in terms of numerical representation. Therefore, the results support the hypothesis of an intricate relationship between spatial representations and numerical representations (DeHevia et al., 2008).

Furthermore, the specific effect of subjective perception of ownership measured in the first three items of PDQ was confirmed in the synchronous condition. The first three items are the ones that consistently assess the feeling of illusion in RHI (Lewis \& Lloyd, 2010). As expected the other items of PDQ were not sensitive to changes across both stroking patterns. Our findings on superior averages for the first three items, specifically in the synchronous stroking, corroborate previous research on ownership illusion that used the same questionnaire format (Lloyd, Gillis, Lewis, Farrell, \& Morrison, 2013; Petkova \& Ehrsson, 2008).

Limitations of the present study include the absence of rigorous experimental control for the magnitude of numerical range between the rulers used to measure the estimates. Though the study respected numerical criteria of increasing number order from left visual field to right visual field in all measurements, it lacked control to a possible effect of the difference on increasing intervals between $0-70 \mathrm{~cm}$ to $70-140$ $\mathrm{cm}$ between the rulers. This research adopted the Mental Number Line hypothesis (Dehaene \& Cohen, 1997) which suggests a compatibility relation between spaces and numerical properties. To that extent the experimental design used increasing rulers from the left visual field to the right visual field. Future research should aim to explore the effects of decreasing rulers from the left to the right, to better evaluate the Mental Number Line hypothesis in the context of proprioceptive recalibration.

A cognitive framework that implies embodiment of outof-body objects will invariably investigate body-space parameters within traditional cognitive processes. In this sense, this paper tried to elucidate how proprioceptive variations can affect fixed numerical space estimations. The main purpose was to elucidate the intertwining between cognitive and multisensory aspects of perception, shedding light onto possible 
cognitive markers of body perception. According to Tsakiris, Schütz-Bosbach and Gallagher (2007), body self-awareness has received special appraisal in the literature over the past two decades because of new technological possibilities on multisensory research. In this scenario, it is interesting to understand how bodily experience establishes itself through the integration between efferent and afferent information of visual and motor action selection of response (Tanaka et al., 2009). Additionally, it is important to understand how these visual and motor selection of responses are connected to symbolic abilities to estimate spaces (Gunderson, Ramirez,

\section{References}

Arend, I., Naparstek, S., \& Henik, A. (2013). Numerical-spatial representation affects spatial coding: Binding errors across the numerical-distance effect. Psychonomic Bulletin \& Review, 20(6), 1181-1186. doi: 10.3758/s13423-013-0428-x

Bekrater-Bodmann, R., Foell, J., Diers, M., Kamping, S., Rance, M., Kirsch, P., . . Flor, H. (2014). The importance of synchrony and temporal order of visual and tactile input for illusory limb ownership experiences an fMRI study applying virtual reality. PLoS One, 9(1), e87013. doi: 10.1371/journal.pone.0087013

Botvinick, M., \& Cohen, J. (1998). Rubber hands 'feel' touch that eyes see. Nature, 391(6669), 756. doi :10.1038/35784

Butz, M. V., Kutter, E. F., \& Lorenz, C. (2014). Rubber hand illusion affects joint angle perception. PLoS One, 9(3), e92854. doi: 10.1371/journal.pone.0092854

Cardinali, L., Brozzoli, C., \& Farnè, A. (2009). Peripersonal space and body schema: Two labels for the same concept? Brain Topography, 21(3-4), 252-260. doi: 10.1007/s10548-009-0092-7

Clayton, H. A., Cressman, E. K., \& Henriques, D. Y. (2013). Proprioceptive sensitivity in Ehlers-Danlos syndrome patients. Experimental Brain Research, 230(3), 311-321. doi: 10.1007/s00221-013-3656-4

Constantini, M., \& Haggard, P. (2007). The rubber hand illusion: Sensitivity and reference frame for body ownership. Consciousness and Cognition, 16(2), 229-240. doi:10.1016/j.concog.2007.01.001

Dehaene, S. (1992). Varieties of numerical abilities. Cognition, 44(1-2), 1-42. doi: 10.1016/0010-0277(92)90049-N

Dehaene, S., \& Cohen, L. (1997). Cerebral pathways for calculation: Double dissociation between rote verbal and quantitative knowledge of arithmetic. Cortex, 33(2), 219-250. doi: 10.1016/S0010-9452(08)70002-9

Dehaene, S., Piazza, M., Pinel, P., \& Cohen, L. (2003). Three parietal circuits for number processing. Cognitive Neuropsychology, 20(3), 487-506. doi: 10.1080/02643290244000239

DeHevia, M. D., Vallar, G., \& Girelli, L. (2008). Visualizing numbers in the mind's eye: The role of visuo-spatial processes in numerical abilities. Neuroscience and Biobehavioral Review, 32(8), 1361-1372. doi: 10.1016/j.neubiorev.2008.05.015

Dempsey-Jones, H., \& Kritikos, A. (2012). Handedness and proprioceptive position estimation: Are left handed people more accurate in self representation and is this representation resistant to manipulation by the rubber hand illusion? Frontiers in Human Neuroscience. Conference Abstract: ACNS-2012 Australasian Cognitive Neuroscience Conference. doi: 10.3389/conf.fnhum.2012.208.00138

Ehrsson, H. H., Spence, C., \& Passingham, R. E. (2004). That's my hand! Activity in premotor cortex reflects feeling of ownership of a limb. Science, 305(5685), 875-877. doi: 10.1126/science.1097011

Ehrsson, H. H., Holmes, N. P., \& Passingham, R. E. (2005). Touching a rubber hand: Feeling of body ownership is associated with activity in multisensory brain areas. Journal of Neuroscience, 25(45), 10564-10573. doi: http://dx.doi.org/10.1523/JNEUROSCI.0800-05.2005

Ehrsson, H. H., Rosén, B., Stockselius, A., Ragnö, C., Köhler, P., \& Lundborg, G. (2008). Upper limb amputees can be induced to experience a rubber hand as their own. Brain, 131, 3443-3452. doi:10.1093/brain/awn297
Beilock, \& Levine, 2012). The Rubber Hand Illusion experiment is an example of manipulating the sense of body selfawareness and ownership that favors the assessment of cognitive and body representations in interaction with environmental cues, allowing the study of volatility and representational cognitive stability in the face of proprioceptive changes. More studies in the field should approach how proprioception and high order cognitive representations are interconnected.

Acknowledgment.- Research financed by CNPq-Brazil.

Fiorio, M., Weise, D., Önal-Hartmann, C., Zeller, D., Tinazzi, M., \& Classen, J. (2011). Impairment of the rubber hand illusion in focal hand dystonia. Brain: A Journal of Neurology, 134(5), 1428-1437. doi:10.1093/brain/awr026

Fiorio, M., Mariotti, C., Panzeri, M., Antonello, E., Classen, J., \& Tinazzi, M. (2014). The role of the cerebellum in dynamic changes of the sense of body ownership: A study in patients with cerebellar degeneration. Journal of Cognitive Neuroscience, 26(4), 712-721. doi:10.1162/jocn_a_00522

Gunderson, E. A., Ramirez, G., Beilock, S. L., \& Levine, S. C. (2012). The relation between spatial skill and early number knowledge: the role of the linear number line. Developmental Psychology, 48(5), 1229-1241. doi: $10.1037 / \mathrm{a} 0027433$

Holmes, K. J., \& Lourenco, S. F. (2012). Orienting numbers in mental space: Horizontal organization trumps vertical. Quarterly Journal of Experimental Psychology, 65(6), 1044-1051. doi 10.1080/17470218.2012.685079

Jones, S. A. H., Cressman, E. K., \& Henriques, D. Y. P. (2010). Proprioceptive localization of the left and right hands. Experimental Brain Research, 204(3), 373-383. doi: 10.1007/s00221-009-2079-8

Kaplan, R. A., Enticott, P. G., Hohwy, J., Castle, D. J., \& Rossell, S. L. (2014). Is body dysmorphic disorder associated with abnormal bodily self-awareness? A study using the rubber hand illusion. PLOS ONE, 9(6), e99981. doi:10.1371/journal.pone.0099981

Lewis, E., \& Lloyd, D. M. (2010). Embodied experience: A first-person investigation of the rubber hand illusion. Phenomenology and the Cognitive Sciences, 9(3), 317-339. doi: 10.1007/s11097-010-9154-2

Lloyd, D. M., Gillis, V., Lewis, E., Farrell, M. J., \& Morrison, I. (2013). Pleasant touch moderates the subjective but not objective aspects of body perception. Frontiers in Behavioral Neuroscience, 7, 207. doi: 10.3389 /fnbeh.2013.00207

Mostafa, A. A., Salomonczyk, D., Cressman, E. K., \& Henriques, D. Y. (2014). Intermanual transfer and proprioceptive recalibration following training with translated visual feedback of the hand. Experimental Brain Research, 232(6), 1639-1651. doi: 10.1007/s00221-014-3833-0

Petkova, V. I., \& Ehrsson, H. H. (2008). If I were you: perceptual illusion of body swapping. PLoS One, 3(12), e3832. doi: 10.1371/journal.pone.0003832

Reuschel, J., Drewing, K., Henriques, D. Y. P., Rösler, F., \& Fiehler, K. (2010). Optimal integration of visual and proprioceptive movement information for the perception of trajectory geometry. Experimental Brain Research, 201(4), 853-862. doi: 10.1007/s00221-009-2099-4

Salomonczyk, D., Cressman, E. K., \& Henriques, D. Y. (2011). Proprioceptive recalibration following prolonged training and increasing distor tions in visuomotor adaptation. Neuropsychologia, 49(11), 3053-3062. doi: 10.1016/j.neuropsychologia.2011.07.006

Shimada, S., Suzuki, T., Yoda, N., \& Hayashi, T. (2014). Relationship between sensitivity to visuotactile temporal discrepancy and the rubber hand illusion. Neuroscience Research, 85, 33-38. doi: 10.1016/j.neures.2014.04.009 
Tanaka, H., Worringham, C., \& Kerr, G. (2009). Contributions of visionproprioception interactions to the estimation of time-varying hand and target locations. Experimental Brain Research, 195(3), 371-382. doi: 10.1007/s00221-009-1798-1

Tsakiris, M., \& Haggard, P. (2005). The rubber hand illusion revisited: Visuo tactile integration and self-attribution. Journal of Experimental Psychology: Human Perception and Performance, 31(1), 80-91. doi: 10.1037/0096-1523.31.1.80
Tsakiris, M., Schütz-Bosbach, S., \& Gallagher, S. (2007). On agency and body ownership: Phenomenological and neurocognitive reflections. Consciousness and Cognition, 16(3), 645-660. doi:10.1016/j.concog.2007.05.012

Van Dijck, J. P., \& Fias, W. (2011). A working memory account for spatialnumerical associations. Cognition, 119(1), 114-119. doi: 10.1016/j.cognition.2010.12.013

(Article received: 15-06-2015; revised: 01-12-2015; accepted: 08-02-2016) 